\title{
APPLICATION OF FINGERTIP SUPPORT TECHNIQUE IN MICROSURGERY
}

\author{
Gergely Csókay ${ }^{1}$, Gergely Pataki², Attila Óvári ${ }^{3}$, István Valálik \\ András Csókay ${ }^{5}$
}

${ }^{1}$ Semmelweis University Department of Maxillo-facial Surgery, Budapest, Hungary

${ }^{2}$ St. Steven Hospital Department of Plastic and Reconstructive Surgery, Budapest, Hungary

${ }^{3}$ Department of Otorhinolaryngology, Head and Neck Surgery, University of Rostock, Germany

${ }^{4}$ St. Johns's Hospital Department of Neurosurgery, Budapest, Hungary

${ }^{5}$ B.-A.-Z. County Hospital Miskolc, Department of Neurosurgery, Miskolc, Hungary csokaya@gmail.com

\section{Abstract \\ Objective}

The physiological tremor which may extend up to $0.4-0.6 \mathrm{~mm}$ on the instrument tip in case of a well-skilled microsurgeon may cause difficulties in any field of microsurgery, in spite of using different armrests. The limit of correctness of medical robots is about $0.1 \mathrm{~mm}$ so far, but the application of these machines is expensive and not convenient for surgeons because the direct touch via microinstruments with living tissues is impossible.

\section{Method}

The effectiveness of the fingertip support technique has been proven by randomized analysis by exact measuring of the reduction of tremor.

\section{Results}

The $0.1 \mathrm{~mm}$ precision could be reached by fingertip support. This extra precise work has not been available by hand so far.

\section{Conclusion}

The significant effect of fingertip support technique in neurosurgery has been certified and published by exact measuring and clinical data.

Our hypothesis could be the indication for trials in any microsurgical work.

Keywords: fingertip support technique; micromanipulation; microsurgery; revascularisation

\section{Introduction}

The precise microsurgical work can be complicated when more than 5-10× magnification is necessary (microneurosurgery, microvascular procedures in plastic and hand surgery, ret- ineal surgery in ophtalmology, cardiac surgery, ear surgery). The most obstructive problem is the physiological tremor which expands up to $0.4-0.6 \mathrm{~mm}$ even in the case of, well skilled microsurgeons us well $3,4,5,6$ (Figure 1). 


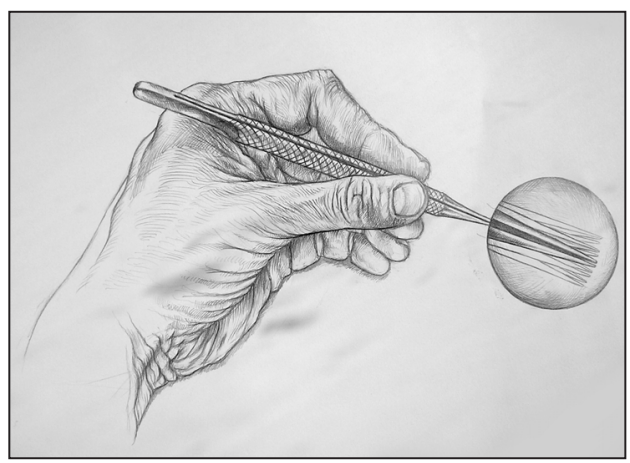

Figure 1. There is a shift caused by the tremor on the end of micro instruments, by the traditional technique

The problems originate from the fact that between the operation point and the last supporting point (end of IV-V fingertip) the carpal joints transmit micro movements (tremors originated neurogenic and mechanical from breathing and heart beat transmitted by circulation to hand) which cause tremors at the end of micro instruments. The fingertip support technique consists of support of I-III fingertip on the crossing bridge (called Bethlehem bridge) above the operating (working) point ${ }^{1,2,3,4}$ (Figure 2).

The end of the bridge rests on the cushions which are fixed on the edge of the exposure. By this technique there are no carpal and finger joints between the last supporting points and the end of instruments which could transmit the tremor. The bridge is necessary because the closest fixed points on the skull or any part of body including the well known armrest devices could be placed so far too far to balance and drive the microinstruments to the operating point only by the fingertip. The fingertip support technique was introduced at different microneurosurgical approaches $1,2,3,4$ (Figure 3) and gives significant reduction of postoperative complications ${ }^{3,4}$.

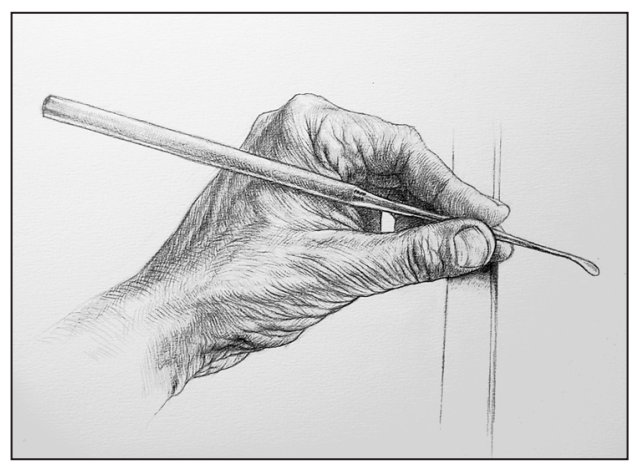

Figure 2. The fingertip support technique reduces the tremor at the end of the instruments

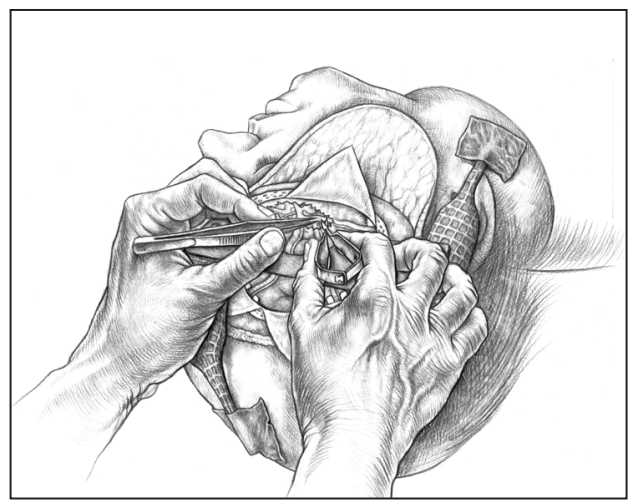

Figure 3. The application of robot hand method in the course of microneurosurgery

Randomized trial of tremor reduction by robot hand technique has not been performed so far. Our hypothesis states that this technique could be introduced in any field of microsurgical approach.

\section{Method}

Effectiveness of the fingertip support technique has been proven by randomized analysis by exact measuring of the reduction of tremor. To measure the difference between the traditional and the new method, we used tracking the instrument tip movements in case of 8 microsurgeons. (3 neurosurgeons, 3 opthal- 
mologists, 1 hand and plastic surgeon, 1 facial and neck surgeon, (aged 30-50), (male and female ratio $(6 / 2)$. The subjects were asked to hold the tip of the instrument above a fixed point with visual control under the microscope with $20 \times$ magnification. At a distance of $5 \mathrm{~mm}$ from the tip of the instrument an adhesive retroreflective marker ( $2 \mathrm{~mm}$ in diameter) was fixed. The position of the marker was registered in a $100 \times 72 \mathrm{~mm}$ field of view in the infrared range with the real-time passive marker-based analyser of movement- $\mathrm{PAM}^{5}$ at a sampling rate of $50 / \mathrm{s}$. Coordinates of the marker positions, evaluated from the images were recorded by a two-dimensional analyzer ${ }^{8}$.

The digital camcorder was positioned on the axis of the forearm of the subject, the elevation angle was approximately perpendicular to the instrument axis. The markers $\mathrm{X}$ and $\mathrm{Y}$ position data then were processed with the MATLAB 7.1 (The Math Works, Inc.). The marker trajectories and displacement data were compared, concerning the two methods of holding the instrument. With each method 3 recording were captured from a subject.

\begin{tabular}{|l|c|c|c|}
\hline & $\begin{array}{c}\text { Traditional } \\
(\mathrm{mm})\end{array}$ & $\begin{array}{c}\text { Traditional } \\
(\mathrm{mm})\end{array}$ & $\mathbf{P}$ \\
\hline $\begin{array}{l}\text { Trajectory field } \\
\text { horizontal }\end{array}$ & $0.53 \pm 0.054$ & $0.15 \pm 0.030$ & 0.000 \\
\hline $\begin{array}{l}\text { Trajectory field } \\
\text { vertical }\end{array}$ & $0.46 \pm 0.044$ & $0.13 \pm 0.020$ & 0.000 \\
\hline $\begin{array}{l}\text { Displacement } \\
\text { RMS }\end{array}$ & $0.36 \pm 0.046$ & $0.10 \pm 0.013$ & 0.001 \\
\hline
\end{tabular}

Table 1. Comparison of measurement data with traditional and fingertip support methods recording of a microsurgeon.

The new method resulted highly significant $(P<0.005)$ reduction of vertical and horizontal trajectory field and root-mean-square (RMS) of displacement

\section{Results}

Comparison of marker trajectories showed a significant regress of trajectory field ranges (Table 1) with the new instrument holding (fingertip support) technique. The significant reduction of tremor $(3,0-4,4)$ was measured at each subject by fingertip support technique.

The displacement intensity, and the mean displacement values showed significant regress. The maximal displacement with the new method improved from $0.61 \mathrm{~mm}$ to $0.27 \mathrm{~mm}$. The most impressive effect was observed in the vertical maximal value with regress from $0.53 \mathrm{~mm}$ to $0.18 \mathrm{~mm}$.

\section{Conclusion}

The significant reduction of tremor (3.0-4.4) was measured at each subject by fingertip support technique, so we stopped the randomized analysis after the 8 person. The microsurgical work one of the hardest manual work in medicine. Unfortunately microsurgical work practice is hardly affordable in most part of the

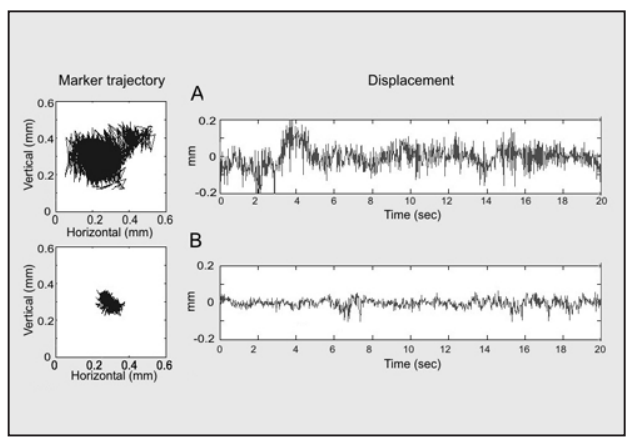

Marker trajectories and displacement curves registered with passive marker based analyser of movements with sampling rate of $50 \mathrm{~Hz}$. The trajectory field and the displacement oscillations with the new method (B) are significantly reduced compared to traditional (A) technique 
world. The continuous practice, peaceful spirit helps the surgeon's hand "to stay quiet" during the operations. Even so the tremor may cause difficulties or damages.

There are trials to introduce the medical robots $^{7,9}$ into microsurgery, but their application has not been spread in the microsurgical practice. The preciseness of the most modern robots are $0,1 \mathrm{~mm}^{9}$. The preciseness the of fingertip support technique reduces the tremor close to $0,1 \mathrm{~mm}$ as well. The manipulation by the surgeons' hand is easier than by the robots.

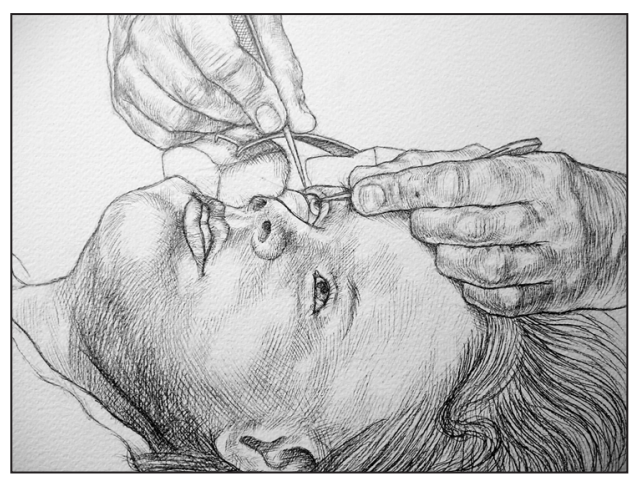

Figure 4. The application of fingertip support method in the course of ophthalmic (retinal) surgery

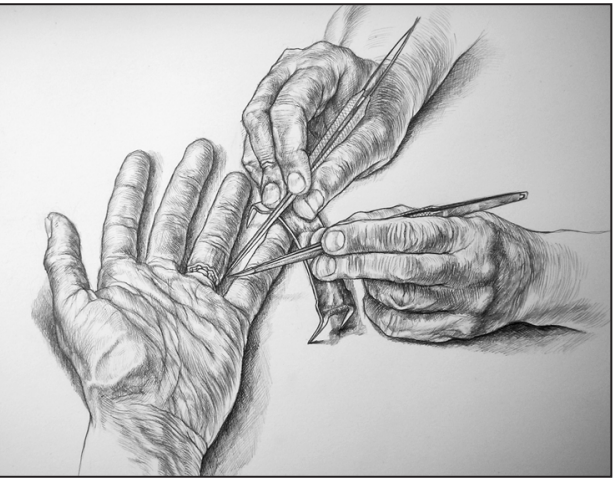

Figure 6. The application of fingertip support method in the course of finger replantation (revascularization in hand surgery)
The contact of instruments with the living tissues is also very important during surgery. The new technique guarantees it. By fingertip support technique the microsurgical procedures could be available to more surgeons to avoid the postoperative complication. Moreover the mental load on the microsurgeon could be reduced. Our hypothesis suggests the introduction of this technique in any other field of microsurgery (Figure 4, 5, 6, 7).

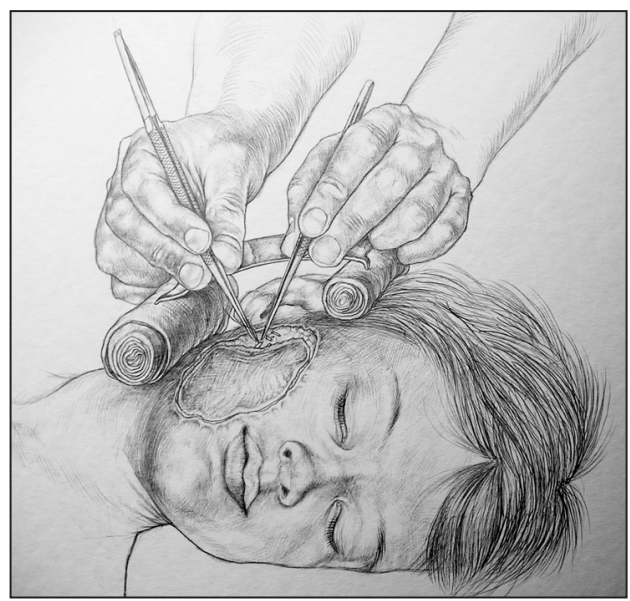

Figure 5. The application of fingertip support method in the course of microrevascularisation of free skin flap transplantation in plastic surgery. The place of Bethlehem bridge is similar in oto surgery (implantation of ear bones)

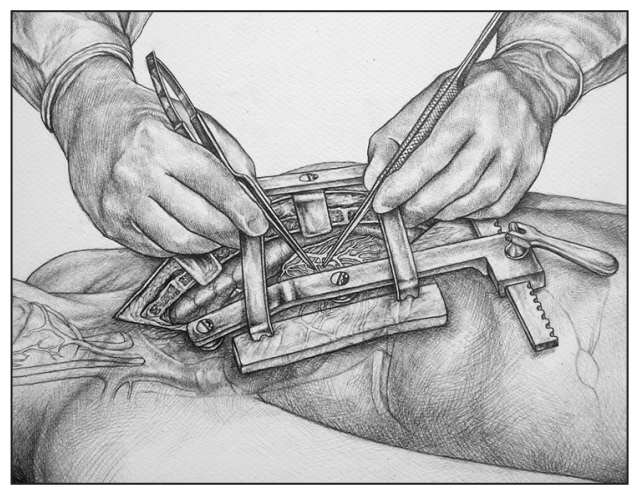

Figure 7. The possible application of robot hand technique in the course of coronary surgery 


\section{REFERENCES}

1. Csókay A, Csókay $G$. Catch fingertip support in microsurgery to reduce the tremor: Technical note (in Hungarian). Hungarian Medical Journal 2006;147:21.

2. Csókay A. A novel microsurgical technique reduces hand tremor in the course of lateral suboccipital approach. Surgical Neurology 2007;67: 392-94.

3. Csókay A, Valálik I, Jobbágy Á. Early experiences with a novel (robot hand) technique in the course of microneurosurgery. Surgical Neurology 2009; 71:469-472.

4. Csókay A, Valálik I, Jobbágy Á. Application of robot hand technique in the course of microneurosurgical operations (in Hungarian). Clinical Neuroscience 2009;62:48-52
5. Ferguson RL, Jobe K. A quiet hand for microneurosurgery: twiddle your thumb. J Neurosurgery 2004;101:541-544.

6. Hartwell RC, Ferguson RL. Physiologic tremor and microsurgery. Microsurgery 1983;4:187-192.

7. Hubschman JP, Bourges JL, Choi W, Mozayan A. "The Microhand": a new concept of micro-forceps for ocular robotic surgery. Eye 2009 Mar 20; S1476-5454

8. Jobbágy Á, Harcos $P$, Károly R. Analysis of fingertapping movement. J Neurosci Methods 2005; 141:29-39.

9. Russel HT, Stoianovici D. Medical Robotics in Computer-Integrated Surgery. IEEE Transactions on Robotics and Automation 2003;19: 765-82.

\section{Special thanks for the medical illustrations to Balázs Sándor Muhari-Papp}

\section{Gergely Csókay}

Semmelweis University Department of Maxillo-facial Surgery, Budapest, Hungary H-1085 Budapest VIII., Üllői út 26. 